\title{
Marker-assisted selection to accelerate forage improvement
}

\author{
B. BARRETT, A. GRIFFITHS, C. MERCER, N. ELLISON, M. FAVILLE, S. EASTON \\ and D.R. WOODFIELD \\ AgResearch Grasslands, Private Bag 11008, Palmerston North \\ brent.barrett@agresearch.co.nz
}

\begin{abstract}
The use of biotechnology, in the form of gene mapping, marker-assisted selection (MAS), and map-based cloning can increase breeding efficiency and the array of traits amenable to selection. The increased efficiency is achieved by combining conventional glasshouse and field evaluations with DNA marker-based selection for superior genes; thereby maximising genetic gain from selection and/or improving traits with low heritability which are not amenable to improvement by conventional breeding alone. Implementation of MAS also reduces costs by permitting selection of superior plants at an early developmental stage or in the absence of the pathogen or pest. Robust genetic linkage maps are the basic requirement for identifying the genes controlling agronomically significant traits, and subsequent implementation of MAS. Recent advances in biotechnology have developed high-throughput, gene-targeted marker systems that allow rapid development of comprehensive genome maps and MAS programmes.

Core linkage maps based on multiple DNA marker technologies are being developed for two of New Zealand's most important forage genomes, white clover (Trifolium repens) and perennial ryegrass (Lolium perenne). Markers from these maps will be used to: a) identify genes controlling simple and complex traits, b) implement targeted MAS strategies, c) transfer novel traits from related species into elite forage cultivars via crosshybridisation, d) clone genes to be inserted directly into elite cultivars, e) provide DNA fingerprints for variety identification, and f) identify putative heterotic groups for hybrid cultivar development.
\end{abstract}

Keywords: DNA markers, EST, gene mapping, linkage, Lolium perenne, marker-assisted selection, Trifolium repens

\section{Introduction}

White clover and perennial ryegrass breeding traditionally involves recurrent selection, a cyclic process which accumulates superior alleles (gene variants) in a population or cultivar. Each cycle requires identification of genetically diverse plants, direct measurement of plants in field and/or glasshouse, accurate selection of superior individuals, and hybridising among selected individuals to create a new set of diverse plants. This process is repeated until enough beneficial alleles have been accumulated, often requiring five or more cycles, with each cycle taking 1-3 years. Although recurrent selection has led to the development of greatly improved forage plant types, it is hampered by laborious phenotypic testing, long cycle times, and environmental effects on trait expression, resulting in slow progress or inability to improve complex traits with low heritability such as nutrient use efficiency or competitive ability. Recurrent selection is also inadequate for identification of new and novel trait types that may exist in elite forage germplasm or related genetic resources.

The convergence of recent advances in biotechnology with these traditional breeding methods has created opportunities in forage improvement. This paper provides an overview of biotechnology as it relates to forage improvement, and describes research in progress to implement gene mapping and marker-assisted selection capability in forage breeding programmes.

\section{Utilising biotechnology for forage improvement}

Genetic linkage maps of forage species have become an essential resource for forage plant breeders. Genetic maps consist of discretely inherited characteristics, called markers, at defined places along each chromosome of a genome. The location of each marker on the genetic map is determined relative to other markers. Although molecular marker maps of some crop species were developed and validated over 15 years ago, map development in forage species has lagged. Genetic maps of ryegrass have only recently been completed (Hayward et al. 1998; Bert et al. 1999), and no complete map of the white clover genome has been reported.

Both ryegrass $(2 n=2 x=14)$ and white clover $(2 n=4 x=32)$ have relatively large, complex genomes, that may hinder identification of genes controlling key traits. Synteny, the conservation of gene and genetic marker order among related species, will facilitate 
accelerated gene mapping and discovery by providing genetic data from species such as the model plant Arabidopsis (a mustard-like weed), the model forage legume Medicago truncatula (barrel medic) and the model grass Oryza sativa (rice) for use in less characterized species, including white clover and perennial ryegrass (Cook 1999; Yuan et al. 2001).

Genetic markers are most commonly DNA-based, but qualitative morphological characters (e.g., incompatibility locus $S$ ) and proteins (e.g., isozymes) also have been used for mapping. DNA markers may be classified based on their source, either anonymous DNA sequences or gene-targeted DNA sequences, such as expressed sequence tags (ESTs), which may be obtained from bioinformatics resources. Although each class is suited to specific applications, integrated use of both anonymous and gene-targeted marker systems is required for comprehensive genome analysis and to compensate for uneven distribution of markers within plant genomes.

Genetic markers can also be classified as either high- or low-throughput. Access to high-throughput, gene-targeted marker technologies such as microsatellites (SSRs), simple nucleotide polymorphisms (SNPs), and sequence-characterized amplified regions (SCARs) is required for creating plant breeding programmes that effectively integrate biotechnology resources. Comprehensive EST databases, containing the DNA sequence of the genes expressed by a specific species such as white clover or perennial ryegrass, are a valuable resource that can be used to develop these high-throughput, gene-targeted markers types.

Gene-targeted DNA markers may also be developed using the known DNA sequences of specific genes identified in other organisms to focus searches for similar genes in forage species. This is especially useful for families of genes in which there are conserved DNA sequence motifs. An example is pest and pathogen resistance (R) genes. DNA sequence data from R genes that have been cloned from a number of species have revealed highly conserved motifs (Williamson 1999; Pan et al. 2000), which may be used to efficiently identify similar sequences in forage genomes.

\section{Marker-assisted selection}

Implementation of the various marker systems will result in comprehensive genetic maps of perennial ryegrass and white clover. These will facilitate identification of genes controlling economically important traits including forage yield, seed yield, persistence, pest and pathogen reaction, forage quality, nutrient use efficiency, and new and novel traits not traditionally associated with forage production. Some traits may be controlled by one major gene, and exhibit qualitative trait characteristics (e.g., susceptible or resistant). For this type of trait, the location of a single gene, such as an R gene, can be identified in individual plants by the presence of closely linked markers from a genetic map. More complex traits, such as forage yield, exhibit continuously distributed trait values and are controlled by several or many genes, termed quantitative trait loci (QTLs). Collection and integration of robust trait and genetic marker data is pivotal for QTL identification, because multiple genes and their individual and combined effects on a trait must be accurately described.

Implementation of MAS strategies in conjunction with phenotypic analysis allows efficient identification of superior progeny. In a MAS programme, a plant breeder uses markers that are linked to major genes or QTLs as signposts to track and select for the gene(s) among segregating progeny. When favourable genes can be more efficiently selected, the length of time from the initiation of a trait-targeted breeding scheme to development of enhanced germplasm can be significantly reduced (Tanksley \& Nelson 1996; Young 1999). A clear example in perennial forages is using MAS evaluation at the seedling stage for a trait normally requiring identification in 2- or 3-year-old plants, thereby reducing the time and space needed to develop plant populations with desired characteristics. If markers are developed from reliable phenotypic data, MAS also minimizes the environmentally induced variation in conventional recurrent selection schemes, which has often confounded efforts to improve complexly inherited traits. Another key advantage of MAS is the ability to determine allele dosage at important loci within individual plants, which facilitates parent plant selection for hybridisation programmes.

\section{Biotechnology for gene discovery and transfer}

Biotechnology also allows plant breeders to capitalise on genetic resources outside the species' immediate gene pool. This may involve genetic mapping and MAS to identify and transfer novel genes into perennial ryegrass or white clover through inter-specific bridge crosses. However, these crosses can be very difficult to achieve. If interspecific crosses cannot be successfully conducted, then markers tightly linked to the genes of interest can provide a springboard for map-based cloning of the gene and transgenic insertion into elite forage cultivars.

\section{Research targets}

Our forage genetic mapping projects in perennial ryegrass and white clover currently are focused on development of the basic genetic marker sets and linkage maps necessary for gene mapping and MAS implementation. This effort encompasses four target areas: 
(a) Development of gene-targeted, high-throughput genetic markers.

(b) Development of robust genetic linkage maps in perennial ryegrass and white clover.

(c) Development of perennial ryegrass and white clover mapping populations suitable for QTL analysis and subsequent MAS.

(d) Identification of genetic markers associated with RKN immunity in Trifolium semipilosum.

\section{Status of forage genome mapping research targets}

\section{Perennial ryegrass}

Molecular markers with high-throughput capacity are being developed for use in perennial ryegrass mapping research. Gene-targeted SSR markers have been identified using the di-, tri-, tetra-, and penta-nucleotide motifs to mine an EST database. This ryegrass EST dataset yielded 1591 putative SSR loci. ESTs with identified SSR motifs were used to develop flanking primer pairs, which were successfully designed for $1304(82 \%)$ of the putative SSRs. A preliminary screening among 9 of 14 SSR primer pairs yielded products of the predicted size, which may be useful as markers for linkage mapping.

A restriction fragment length polymorphism (RFLP) survey among perennial ryegrass pair cross parents, utilising probes derived from a perennial ryegrass cDNA library, was used to select an optimal pair cross for use as a mapping population. Parents ('A6595' (a Southland ecotype) and 'Ruanui') and $171 \mathrm{~F}_{1}$ progeny from this pair cross are being used for linkage analysis. Initially a subset $(n=45)$ of the population is being used for RFLP mapping. Key traits segregating in this perennial ryegrass population include flowering time, cold and drought tolerance, shoot and root morphology, leaf shear strength, and soluble carbohydrate content.

\section{White clover}

A white clover EST database has been used to develop gene-targeted SSRs. Initial analysis has yielded a similar number of primer pairs as was detected in the ryegrass EST dataset. These putative high-throughput markers will be validated and used for white clover genetic linkage mapping, QTL identification, gene discovery, and subsequent MAS programmes.

Ninety-one $F_{1}$ progeny from a pair cross between an elite white clover plant developed in New Zealand and one from the United States are being utilised to develop a framework genetic linkage map. To date, 208 single-dose RFLP and 81 amplified fragment length polymorphism (AFLP) markers have been identified according to methods described by Wu et al. (1992).
Pending acquisition of additional marker data, linkage analysis will be conducted to integrate genetic markers from both parents into a single reference map consisting of 16 marker-saturated linkage groups.

The convergence of the white clover high-throughput genetic marker and framework map development goals outlined above will facilitate further mapping efforts to identify QTLs influencing key agronomic traits, and utilisation of MAS to develop superior forage cultivars for use in New Zealand agriculture. One example of this process is the development of nematode resistant white clover cultivars. Plant-parasitic nematode infestations annually destroy NZ\$240 billion worth of global agricultural production (Williamson 1999). In New Zealand, the nematode-induced reduction in clover yield results in an annual loss of NZ\$1 billion in animal production (Mercer et al. 2000). Current efforts to develop resistant cultivars are hampered by environmental effects on phenotypic evaluation, the multigenic nature of the resistance in some germplasm, and the length of time required for each cycle of recurrent selection. Mapping of the gene(s) conferring nematode resistance in white clover and implementation of MAS will expedite transfer of nematode resistance from agronomically inferior resistant plant types into agronomically superior, marketable clover cultivars.

In the 1980s, two independent recurrent selection programmes to develop white clover plants resistant to the clover root-knot nematode (RKN; Meloidogyne trifoliophila) and to the clover cyst nematode $(\mathrm{CCN}$; Heterodera trifolii) were initiated. Using classical forage breeding methodology, up to seven cycles of recurrent selection have developed plants with moderate and high levels of resistance to RKN and $\mathrm{CCN}$, respectively (Mercer et al. 1999; 2000). These resistances have also been combined in the same genetic background (Mercer \& Miller 2000). Although many of these resistant plants are inferior when compared to elite cultivars; they are an excellent genetic resource for genome mapping and identification of markers linked to the $\mathrm{R}$ gene(s) conferring the resistant phenotype.

An $F_{1}$ population developed from a pair cross between 'Sustain' and a nematode resistant plant were evaluated for CCN and RKN reaction in glasshouse assays during March and April 2001. This screening has resulted in identification of a normal distribution of RKN reaction phenotypes; slightly skewed toward the resistance phenotype (Figure 1); confirming the complex inheritance reported by Mercer et al. (2000). The phenotypic data will be integrated with DNA marker genotypes derived from this population to identify QTLs involved in this resistance; and to begin implementing MAS strategies for developing elite; 
nematode resistant white clover cultivars. Field trials also will be established in the 2000/2001 growing season in the Manawatu and Canterbury districts to initiate identification and mapping of gene(s) controlling other economically significant characteristics.

\section{Gene discovery using biotechnology}

Introduction of novel sources of pathogen resistance into white clover will contribute to sustainability of forage production. An example is the utilisation of a diploid near relative of white clover; Trifolium semipilosum $(2 \mathrm{n}=2 \mathrm{x}=16)$; as a source of $\mathrm{RKN}$ immunity. Despite extensive $T$. repens germplasm surveys and recurrent selection for resistance; white clover genotypes with immunity to the RKN have not been identified. Alternatively; immune and susceptible $T$. semipilosum plants have been identified (Mercer \& Grant 1993). On the basis of RKN reaction phenotype; bulked DNA samples; either immune or susceptible; were used to screen for AFLP marker polymorphism associated with the immune phenotype. Twenty-nine polymorphisms associated with the immune phenotype were identified by screening these bulks with 40 primer combinations (Figure 2). Evaluation of these polymorphic markers in an $F_{1}$ mapping population derived from a paired cross between a resistant and a susceptible individual will allow us to quantify linkage intensity between these markers and the $\mathrm{R}$ gene(s) conferring the immunity. Tightly linked AFLP markers will be converted into high-throughput SCAR markers and used for MAS to transfer the trait into agronomically superior white clover germplasm; or for high resolution mapping to isolate the $\mathrm{R}$ gene from $T$. semipilosum if cross hybridisation efforts fail.

Identification of RKN immunity in T. semipilosum is only one of many gene-discovery opportunities within the Margot Forde Forage Germplasm Centre; a major international gene bank storing seed samples representing over 1600 temperate forage species from 83 countries. Utilisation of these germplasm resources in conjunction with new biotechnology tools will facilitate discovery of genes improving traditional forage characters; as well as delivery of new and novel traits not traditionally associated with forage cultivars.

\section{Conclusion}

Access to novel germplasm and an array of capabilities provides a strong platform to integrate biotechnology and conventional forage improvement. Initial datasets and preliminary analyses suggest that genetic maps of perennial ryegrass and white clover will be achieved in the near future. Emphasis on development of genetargeted markers amenable to high-throughput analysis
Figure $₫ 1$ The frequency distribution of disease intensity score among white clover $F_{1}$ progeny $(n=182)$, following inoculation with the clover root-knot nematode (Meloidogyne trifoliophila). Seedlings were inoculated with 3000 eggs each at 3 weeks after germination. Root galling (indicative of infection) was scored 21 days later. Data are scored on a scale of 0 to $5 ; 0=$ no galls, $1=$ $20 \%$ galling, $2=40 \%$ galling, $3=60 \%$ galling, $4=80 \%$ galling, $5=100 \%$ galling.

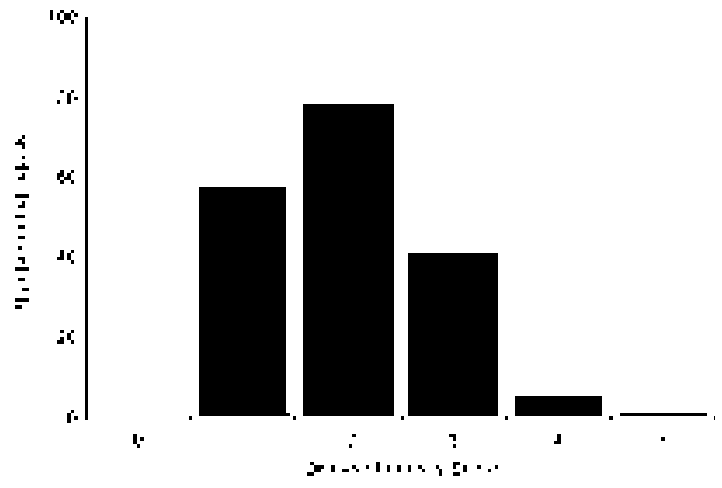

Figure $\llbracket 2$ A DNA marker associated with nematode immunity. AFLP primer pair Pstl-at:Msel-ct was screened against immune (I) and susceptible (S) Trifolium semipilosum genotypes. Bulk samples were created from DNA extracts of 10,20 , or 30 individual seedlings exhibiting qualitative I or $S$ reactions after inoculation with the clover-root knot nematode, Meloidogyne trifoliophila. Lane 'M' contains size standard DNA, sizes are base pairs.

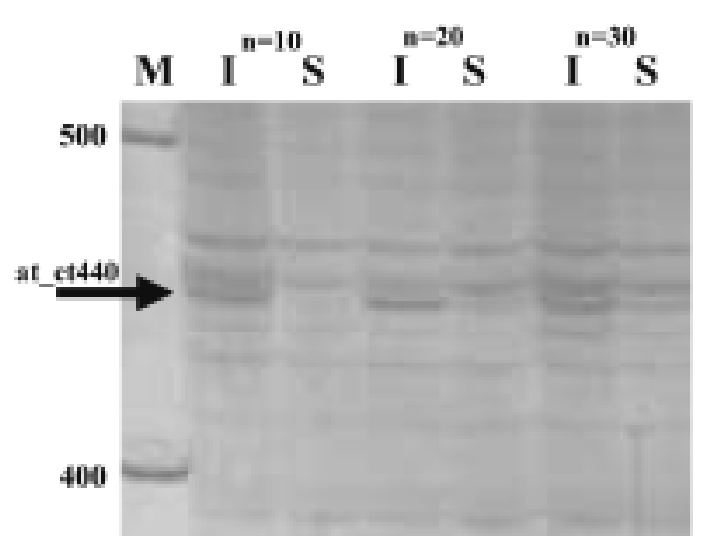

will facilitate our transition from conventional breeding efforts into biotechnology-integrated forage improvement programmes; and provide a capability for map-based cloning of genes controlling key traits. Implementation of MAS capabilities for development of enhanced cultivars; including white clover with 
durable nematode resistance; will be commenced upon completion of the initial mapping and marker development projects.

\section{ACKNOWLEDGEMENTS}

Mark Schreiber conducted EST database analysis and SSR primer design. Greig Cousins; Benjamin Franzmayr; and Kenyon Moore provided technical assistance for the white clover research. Funding was provided by the Foundation for Research Science and Technology.

\section{REFERENCES}

Bert; P.F.; Charmet; G.; Sourdille; P.; Hayward; M.D.; Balfourier; F. 1999. A high-density molecular map for ryegrass (Lolium perenne) using AFLP markers. Theoretical and Applied Genetics 99: 445-452.

Cook; D.R. 1999. Medicago truncatula - a model in the making! Current Opinion in Plant Biology 2: 301-304.

Hayward; M.D.; Forster; J.W.; Jones; J.G.; Dolstra, O.; Evans, C.; McAdam, N.J.; Hossain, K.G.; Stammers, M.; Will, J.; Humphreys, M.O.; Evans, G.M. 1998. Genetic analysis of Lolium. I. Identification of linkage groups and the establishment of a genetic map. Plant Breeding 117: 451455.

Mercer, C.F.; Grant, J.L. 1993. The development of Meloidogyne hapla (Nematoda: Tylenchida) in resistant and susceptible Trifolium semipilosum. Proceedings of the $6^{\text {th }}$ Australasian Grassland Invertebrate Ecology Conference: 195-201.

Mercer, C.F.; van den Bosch, J; Miller, K. 1999. Effectiveness of recurrent selection of white clover (Trifolium repens) for resistance to New Zealand populations of clover cyst nematode (Heterodera trifolii). Nematology 1: 449-455.

Mercer, C.F.; Miller, K.J. 2000. Combining resistance in white clover to root-knot and clover cyst nematodes. Proceedings of the New Zealand Society for Parasitology (Abstr.) (in press).

Mercer, C.F.; van den Bosch, J.; Miller, K. 2000. Progress in recurrent selection and in crossing cultivars with white clover resistant to the clover root-knot nematode Meloidogyne trifoliophila. New Zealand Journal of Agricultural Research 43: 4148.

Pan, Q.; Wendel, J.; Fluhr, R. 2000. Divergent evolution of plant NBS-LRR resistance gene homologues in dicot and cereal genomes. Journal of Molecular Evolution 50: 203-213.

Tanksley, S.D.; Nelson, J.C. 1996. Advanced backcross QTL analysis: a method for simultaneous discovery and transfer of valuable QTLs from unadapted germplasm into elite breeding lines. Theoretical and Applied Genetics 92: 191-203.

Williamson, V.M. 1999. Plant nematode resistance genes. Current Opinion in Plant Biology 2: 327331.

Wu, K.K.; Burnquist, W.; Sorrells, M.E.; Tew, T.L.; Moore, P.H.; Tanksley, S.D. 1992. The detection and estimation of linkage in polyploids using singledose restriction fragments. Theoretical and Applied Genetics 83: 294-300.

Young, N.D. 1999. A cautiously optimistic view for marker-assisted breeding. Molecular Breeding 5: 505-510.

Yuan, Q.; Quackenbush, J.; Sultana, R.; Pertea, M.; Salzberg, S.L.; Buell, C.R. 2001. Rice bioinformatics: analysis of rice sequence data and leveraging the data to other plant species. Plant Physiology 125: 1166-1174. 